\title{
Modelación del agua subterránea en plantaciones de palma de aceite y pastizales mediante técnicas geofísicas
}

\section{Modelation of groundwater in oil palm plantations and grasslands across geophysic technics}

\begin{abstract}
María Dolores Sánchez-García [D, Jonathan Bolaina-Vazconcelos 1 [D] Guillermo Chávez-Hernández ${ }^{1 *}$ (D) Daniel Andrés Damas-López ${ }^{1}$ [D Maximiano Antonio EstradaBotello2

Juan de Dios Mendoza-Palacios² Rufo Sánchez-Hernández 2 [D

${ }^{1}$ División Académica de Ciencias Básicas. Universidad Juárez Autónoma de Tabasco. Carretera Cunduacán-Jalpa de Méndez, Km. 1. CP. 86690 Cunduacán, Tabasco, México.

${ }^{2}$ División Académica de Ciencias Agropecuarias. Universidad Juárez Autónoma de Tabasco. Carretera Villahermosa-Teapa Km. $25 \mathrm{R} / \mathrm{A}$ La Huasteca, Segunda Sección. CP. 86280. Villahermosa, Tabasco, México.

*Autor de correspondencia:
\end{abstract} guillermo.chavez@ujat.mx

Artículo científico

Recibido: 26 de febrero 2021

Aceptado: 08 de octubre 2021

Como citar: Sánchez-García MD, Bolaina-Vazconcelos J, ChávezHernández G, Damas-López DA, Estrada-Botello MA, MendozaPalacios JD, Sánchez-Hernández R (2021) Modelación del agua subterránea en plantaciones de palma de aceite y pastizales mediante técnicas geofísicas. Ecosistemas y Recursos Agropecuarios 8(3): e2901. DOI: 10.19136/era.a8n3.2901
RESUMEN. Se realizó una investigación durante la temporada de lluvias, con el objetivo de caracterizar mediante tomografía de resistividad eléctrica (TRE), la distribución del agua subterránea en tres áreas cultivadas con palma de aceite (Elaeis guineensis Jacq.) de 5, 11 y 25 años de edad, y se compararon con pastizales aledaños. En cada área se realizaron 5 perfiles con arreglo dipolo-dipolo en dirección NE-SO, que abarcaron 40 metros de pastizal y 80 metros dentro de las plantaciones, con separación electródica de 5 metros, y 20 metros entre perfiles. A través de un resistivímetro Syscal Junior de IRIS Instrument, se calculó la distribución de la resistividad eléctrica de un gran número de medidas de resistividad aparente del subsuelo, que permitió generar una imagen eléctrica que muestra la distribución de la resistividad verdadera del subsuelo. En cada línea se realizó la inversión de datos a través del software Res2Dinv, para generar un modelo 2D, donde se observa un patrón de resistividad eléctrica de mayor a menor según la profundidad del terreno, lo que supone la presencia arenas, limos, aluviones, arcillas y gravas en toda el área. El nivel de la zona saturada es más profundo en las plantaciones de palma con respecto al pastizal, particularmente en plantaciones de 25 años. A pesar de las lluvias de la temporada, la zona de saturación en el subsuelo es más profunda en plantaciones de palma de aceite que en pastizales, lo que sugiere que el cultivo abate más agua que los pastizales aledaños.

Palabras clave: Elaeis guineensis Jacq., hidrología, resistividad eléctrica, sustentabilidad, trópico húmedo.

ABSTRACT. A research was carried out during the rainy season, with the aim of characterizing through electrical resistivity tomography (ERT), the distribution of groundwater in three areas cultivated with oil palm (Elaeis guineensis Jacq.) of 5, 11 and 25 years old, and were compared respect to grassland adjacent. In each area, 5 profiles were made with a dipole-dipole arrangement in a NE-SO direction, covering 40 meters of grassland and 80 meters within the plantations, with electrodic separation of 5 meters, and 20 meters between profiles. Through an IRIS Instrument Syscal Junior resistivity meter, the distribution of electrical resistivity was calculated from a large number of measurements of subsoil apparent resistivities, that allowed to generate an electrical image that shows the distribution of the true resistivity of the subsoil. In each line, the data inversion was carried out through the Res2Dinv software, to generate a $2 \mathrm{D}$ model, where an electrical resistivity pattern is observed from highest to lowest depending on the depth of the ground, which implies the presence of sands, silts, alluvium, clays and gravel in throughout the area. The level of the saturated zone is deeper in oil palm plantations than in grasslands, particularly in plantations of 25 years old. It is concluded that, despite the seasonal rains, the saturation zone in the subsoil is deeper in oil palm plantations than in grasslands, which suggests that the crop can remove more water than the adjacent grasslands.

Key words: Elaeis guineensis Jacq., hydrology, electric resistivity, sustainability, humid tropic. 


\section{INTRODUCCIÓN}

La superficie del cultivo de palma de aceite (Elaeis guineensis Jacq.) ha crecido en aproximadamente $30 \%$ durante la última década en el sureste mexicano, al pasar de 30034.77 ha en el 2007 a 101753.22 ha en el 2018 (SIAP 2019). Esta expansión ha provocado controversias con relación a los impactos sociales y ambientales que trae consigo su cultivo (Isaac-Márquez et al. 2016). De acuerdo con Mingorría (2018), los cultivos de palma de aceite requieren de grandes cantidades de agua para ser altamente productivos, por lo que cualquier factor que incida en la cantidad y calidad del agua subterránea no sólo repercute en el propio estado de los acuíferos, sino también en el mantenimiento y equilibrio de los ecosistemas naturales que directa o indirectamente dependen del agua subterránea. Una de las razones por las cuales se ha expandido el cultivo de palma de aceite en el sur de México, es la alta disponibilidad de lluvia, la cual llega acumular entre 2500 y 3000 mm al año (Ruiz-Álvarez et al. 2012). De acuerdo a la pluviometría, en Tabasco se identifica una época de nortes, que se caracteriza por aumento de humedad, y reducciones de precipitación, temperatura, radiación y evapotranspiración; de secas con precipitaciones escasas, presencia de vientos secos y elevadas temperaturas, evaporación y radiación, y de lluvias, con incrementos de precipitación y presencia de una canícula (Maldonado et al. 2006). Por ello, los estudios del régimen hídrico, la morfología general e inventario hídrico, son necesarios para determinar las características de las fuentes, su extensión y calidad del agua para su utilización y control, así como para determinar la disponibilidad en cantidad y calidad en un determinado lugar o período de tiempo, para satisfacer las demandas identificables, ya sean para consumo humano, agrícola, energético u otros (Sellers et al. 2015).

Para Chia et al. (2020) una salida de agua del acuífero es la transpiración, fenómeno fisiológico que permite la captación del agua por las raíces, el transporte por el sistema vascular, hasta su liberación hacia la atmosfera por las hojas; cuando se suma al agua que se pierde por evaporación, al proceso se le denomina evapotranspiración. En el caso particular de las plantaciones de palma de aceite, Yusop et al. (2008), determinaron que contrario a lo que se piensa, las plantaciones menores a dos años, pueden transpirar entre 1365 y $1405 \mathrm{~mm}$ año $^{-1}$, una cantidad mayor a la de las plantaciones de nueve años, que es alrededor de 927 a 1098 $\mathrm{mm}$ año ${ }^{-1}$, aunque no descartan que esta diferencia, puede deberse a una subestimación en plantaciones de mayor edad, de manera general concluyeron que la evapotranspiración en los cultivos de palma de aceite, son similares a la de los bosques tropicales, que es de 1000 a $1300 \mathrm{~mm}$ año ${ }^{-1}$. La infiltración constituye una entrada o recarga al acuífero, esta depende de la textura y usos del suelo, por lo que la infiltración depende de la intensidad y cantidad de lluvia, de las propiedades edáficas y de las interacciones entre ellas, a este gradiente de penetrabilidad se le denomina conductividad hidráulica (Noguchi et al. 1997a). Aparte de los factores descritos, la infiltración también varía dentro de una zona de cultivo, en el caso de la palma de aceite, la tasa de infiltración es distinta entre las zonas debajo de la palma, el camino de cosecha y las franjas donde se deposita la fronda o montículo de hojas de residuos, lo que se debe a factores asociados a la macro porosidad que genera la acumulación de la materia orgánica, y el pisoteo de maquinaria y personal durante el manejo del cultivo (Banabas et al. 2008).

Actualmente, la gestión del agua subterránea en México es poco práctica, pues aún no se tienen los mecanismos eficientes para conocer su disponibilidad en los acuíferos, volumen de extracción y grado de contaminación, en su lugar. Al respecto, la Comisión Nacional del Agua (CONAGUA) sugiere el uso de métodos indirectos que permitan calcular los volúmenes de infiltración del agua subterránea (Salazar 2018), dentro de dichos métodos se encuentran los de exploración geofísica, en especial los métodos geoeléctricos, los cuales son en una herramienta fundamental para el estudio de zonas hidrogeológicas. Los sondeos eléctricos verticales (SEV) constituyen un método de exploración rápido, efectivo y económico para la caracterización de una zona, mientras que la tomografía de resistividad eléc- 
trica (TRE) también ha mostrado un incremento en su utilización en los últimos años, ya que ofrece la ventaja de obtener perfiles de resistividad continuos a partir del desarrollo de potentes programas de inversión (Ellis y Oldenburg 1994, Loke et al. 2013), también permite caracterizar acuíferos a partir de las resistividades obtenidas relacionando estas con los parámetros hidráulicos y observar heterogeneidades y anisotropías de este (Slater et al. 2007, Niwas et al. 2011, Perdomo et al. 2014). Aun cuando se han realizado estudios para comprender el impacto del cultivo de palma de aceite a los procesos hidrológicos dentro de las plantaciones de palma de aceite, aun no se comprende del todo, por lo que existe un vacío de información sobre cómo las prácticas agrícolas afectan las funciones hidrológicas terrestres y la calidad del agua en los ecosistemas (Ah Tung et al. 2009). Por la importancia que tiene el cultivo de palma de aceite en el estado de Tabasco, es necesario conocer los impactos que esta especie tiene sobre el ciclo hidrológico del agua, por lo que el objetivo del presente trabajo fue caracterizar las condiciones de la distribución del agua subterránea que subyace en suelos cultivados con palma de aceite de diferentes edades, y compararla con respecto a su condición de uso anterior como pastizal, a través del uso de la tomografía de resistividad eléctrica (TRE) durante el periodo de lluvias.

\section{MATERIALES Y MÉTODOS}

\section{Descripción del área de estudio}

Con base en el geo posicionamiento in situ con equipo portátil GPS marca Garmin ${ }^{\circledR}$, modelo GPSMAP ${ }^{\circledR}$ 62s, el área de estudio se encuentra ubicada en la ranchería Chipilinar tercera sección, del municipio de Jalapa, Tabasco, en las coordenadas geográficas $17^{\circ} 46^{\prime} 52.8^{\prime \prime}$ LN; $92^{\circ} 46^{\prime}$ 05.4" LO, a una altura promedio de 20 metros sobre el nivel del mar. La precipitación promedio anual es de $3783 \mathrm{~mm}$, con promedio máximo mensual de $728 \mathrm{~mm}$ en el mes de septiembre y un promedio mínimo mensual de $81 \mathrm{~mm}$ en el mes de abril (CONAGUA 2020).

El municipio de Jalapa, Tabasco y específicamente, el área total de la zona de estudio, se en- cuentra en una zona de contacto geológico entre aluvión y depósito lacustre del Cuaternario, con los pisos Pleistoceno y Holoceno que dieron lugar al depósito de sedimentos como arenas y limos, materiales de ambiente aluvial, lacustre y palustre. Se pueden encontrar variaciones desde arenisca-lutita en superficie como lutitas-conglomerados polimíctico en profundidad (Zavala-Cruz et al. 2016)

El acuífero La Sierra, se localiza en la porción centro-sur del estado de Tabasco, tiene una superficie de $1817 \mathrm{~km}^{2}$, que cubre los municipios de Teapa, Tacotalpa, Jalapa y, parcialmente los de Macuspana y el Centro. Los principales centros de población localizados dentro del área son las cabeceras municipales de Teapa, Tacotalpa y Jalapa. Limita al norte con el acuífero Samaria-Cunduacán, al sur y este con el estado de Chiapas y al oeste con el acuífero Reforma del estado de Chiapas (CONAGUA 2020).

\section{Tratamientos y muestreo de variables edáficas}

Con base en las edades de las plantaciones, sobre una superficie de 11.23 hectáreas, se ubicaron cuatro áreas de observación, que correspondieron a cuatro tratamientos: Tratamiento 1, consistió en una plantación joven de palma de aceite menor a 5 años de edad (2.41 ha), tratamiento 2, consistió en una plantación en plenitud de producción de 11 años de edad (2.2 ha), tratamiento 3, correspondió a una plantación vieja de más de 25 años de edad, casi en etapa de renovación (falta superficie de esta plantación), y como referencia se consideró el tratamiento 4, que fue pastizal aledaño a las plantaciones (3.85 ha), por tratarse del uso de suelo anterior a la plantación de palma de aceite.

\section{Tomografía de resistividad eléctrica (TRE)}

En el área del polígono se distribuye tanto cobertura de pastizales como de cultivo de palma de aceite de las tres edades. La superficie cultivada con palma de aceite se dividió en tres zonas, cada una de ellas corresponde a cada edad 0 tratamiento: tratamiento 1: plantación de 5 años (Zona 2); tratamiento 2: plantación de 11 años (Zona 1), y tratamiento 3: plantación de 25 años (Zona 3). 
Con la finalidad de que parte de la longitud de cada perfil de las TRE se ubicaran dentro del pasti$z a l, y$ los metros restantes dentro de las plantaciones de palma de aceite, los 15 perfiles se trazaron en dirección NE-SO (Figura 1), 5 perfiles con arreglo dipolo-dipolo por cada zona con separación electródica de $5 \mathrm{~m}$, abarcando una longitud total de 120 m por perfil y separación entre perfiles de $20 \mathrm{~m}$, con excepción del perfil 5 de la zona 2 , donde se tuvo que separar a una distancia de $30 \mathrm{~m}$ debido a un pequeño cuerpo de agua en superficie.

Con base en el arreglo dipolo-dipolo de los perfiles, la profundidad media de investigación $\left(z_{e}\right)$ se calculó a una profundidad de estudio de $12 \mathrm{~m}$. Dicho calculo, se basa en la longitud total del arreglo (L) y un factor geométrico específico para el arreglo antes mencionado. Con el valor de la corriente (I) y del potencial (V) medidos en campo, junto con un parámetro (K) que depende de la distancia entre los electrodos, se calcula la resistividad aparente $(\rho \alpha)$, la resistencia que presenta el subsuelo a que fluya la energía eléctrica, correspondiente a diferentes profundidades. La expresión para el cálculo de la resistividad empleando el arreglo Dipolo-Dipolo es:

$$
\begin{gathered}
\rho \alpha=\pi(n)(n+1)(n+2) \alpha \frac{\Delta}{I} \\
K=\pi(n)(n+1)(n+2) \alpha
\end{gathered}
$$

Dónde: $\mathrm{K}$ es el factor de penetración o coeficiente del dispositivo y $\mathrm{n}$ es el nivel de atribución que corresponde a la separación de los electrodos.

El procedimiento descrito desarrollados por Loke (2000), da idea de la profundidad a la cual será capaz de llegar un arreglo particular, a partir de las profundidades de penetración media para diferentes arreglos, aunque que es válida para modelos homogéneos de la tierra. La tomografía de resistividad eléctrica (TRE), es un método para calcular la distribución de la resistividad eléctrica (RE) en el subsuelo de un gran número de medidas de resistividad aparente, hechas con electrodos posicionados en un patrón geométrico adaptado y diseñado con base al objetivo del estudio, que permite determinar una imagen eléctrica que muestre la distribución de la resistividad verdadera en el subsuelo (TejeroAndrade et al. 2015). Dicho valor se calcula individualmente mediante la inyección de corriente de intensidad conocida en dos electrodos (A y B), y la medición de la diferencia de potencial derivado en otros dos electrodos ( $\mathrm{M}$ y N). En esta primera fase se utilizó un resistivímetro Syscal Junior de IRIS Instrument, con 24 electrodos distribuidos regularmente cada $5 \mathrm{~m}$ a lo largo de una línea recta, con una configuración geométrica tipo dipolo-dipolo, debido a que es una configuración más sensitiva a cambios de resistividad horizontal respecto a otros, por lo que se generó, un total de 15 líneas, cubriendo el área total del polígono de estudio, en dicho polígono se incluyen plantaciones de 5,11 y 25 años de edad respectivamente, así como de pastizales aledaños a las plantaciones. Por cada edad de plantación se instalaron 5 líneas, las cuales iniciaron en el área de pastizal y se prolongaron por $40 \mathrm{~m}$, posteriormente las líneas se internaron $80 \mathrm{~m}$ dentro de las plantaciones de palma, para hacer una longitud total de 120 m por cada línea. La toma de datos se llevó a cabo durante la temporada de lluvias en el mes de septiembre de 2020. El estudio representa uno de los primeros en el estado, en los que se aplican técnicas geoeléctricas para comprender el efecto de un cultivo sobre la dinámica de la zona saturada subyacente, por lo que este representa una base, para investigaciones futuras en épocas de escasa precipitación.

\section{Procesamiento y modelado de datos}

La segunda fase del método se realizó en gabinete, consistió en la inversión de datos por separado en cada una de las líneas, eliminando posibles datos anómalos y obteniendo el menor error posible en las iteraciones sobre las cuales se basa el modelo de resistividad real $(R R)$ del terreno, obtenidas a partir de los registros de campo mediante el software Res2Dinv para un modelo 2D, para la delimitación y caracterización de la zona de recarga, finalmente se interpretaron los datos y se propuso la caracterización de la zona de recarga en el área de estudio, y se asoció este modelo a los tratamientos y los datos obtenidos en las TRE. 


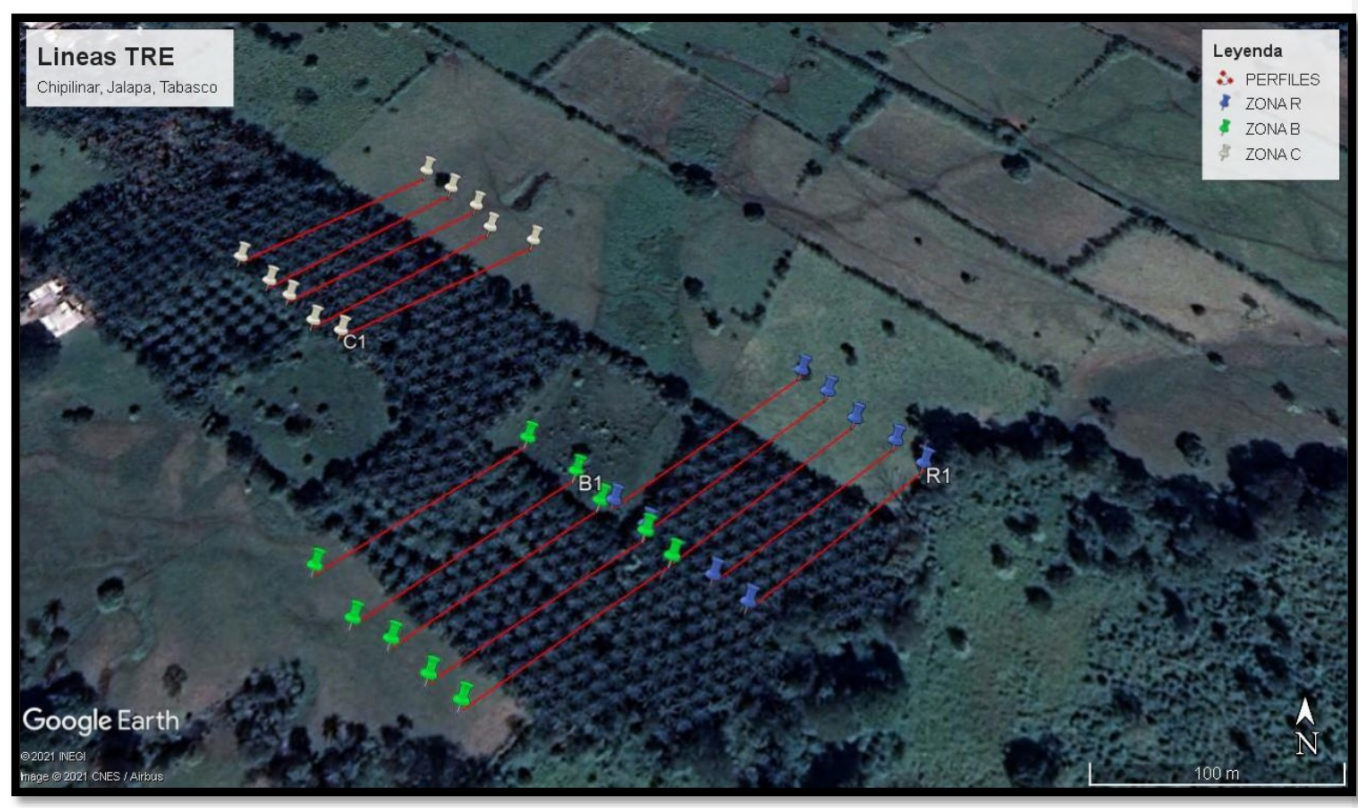

Figura 1. Líneas de la TRE ubicadas en el polígono del área de estudio.

\section{RESULTADOS}

\section{Interpretación de resultados de TRE}

La Figura 2, muestra el resultado de la inversión de los datos de la zona 1, que corresponde a la plantación de palma de aceite de 11 años de edad. En los 5 perfiles obtenidos, los primeros $40 \mathrm{~m}$ de la parte NE fueron obtenidos sobre pastizales, los 80 $\mathrm{m}$ restantes sobre la zona cultivada con palma de aceite. En todos los perfiles, se muestran a través de líneas punteadas, los valores de resistividades bajas, que representan el límite superior de la zona saturada, la cual son las de mayor interés debido a que indican la posible zona de abastecimiento de agua para el cultivo. En el caso de la plantación de 11 años, esta zona saturada se ubica aproximadamente a los $4 \mathrm{~m}$ de profundidad, y se observa que ese nivel se mantiene constante en cada uno de ellos. En los perfiles de esta zona se registraron resistividades que oscilan entre 100 a $800 \Omega \cdot m$; se observa otro rango de resistividades medias de entre 35-100 $\Omega \cdot m$, y resistividades más bajas, que se ubican entre 1-35 $\Omega \cdot m$. Se observa que en los perfiles 2 y 3 , los rangos de resistividad pueden ser superiores a $700 \Omega \cdot m$, en los perfiles 4 y 5 las resistividades alcanzan entre
350-460 $\Omega \cdot m$, y en el perfil 1 el valor máximo de resistividad es aproximadamente de $160 \Omega \cdot m$.

La Figura 3, muestra el resultado de la inversión de datos de la TRE de los 5 perfiles de la zona 2, que corresponde a la plantación de 5 años. En esta zona, las líneas se orientaron en dirección NE-SO los últimos $30 \mathrm{~m}$ de la parte $\mathrm{SO}$ fueron obtenidos sobre pastizales y el resto de los perfiles estaban posicionados sobre la zona del cultivo de la palma de aceite. A diferencia de la zona anterior, en esta, los valores de resistividades más altas oscilan entre 140 a $260 \Omega \cdot m$, las resistividades medias se encuentran en el rango de 35 a $140 \Omega \cdot m$, mientras que las resistividades bajas, oscilan entre 1 a $35 \Omega \cdot m$. Se puede observar que las resistividades más bajas, inician a una profundidad muy somera hacia el SO de los perfiles, donde se encuentran los pastizales y se incrementa la profundidad, hasta los $9 \mathrm{~m}$ hacia el NE, donde se ubica el cultivo de palma de aceite. La presencia de esta unidad de resistividades someras, puede deberse a que en esta zona había encharcamiento de agua de lluvia y se presenta infiltración, lo cual actúa como posible recarga para el suministro de agua para el cultivo y el pastizal presente en la zona.

La Figura 4, muestra el resultado de la in- 


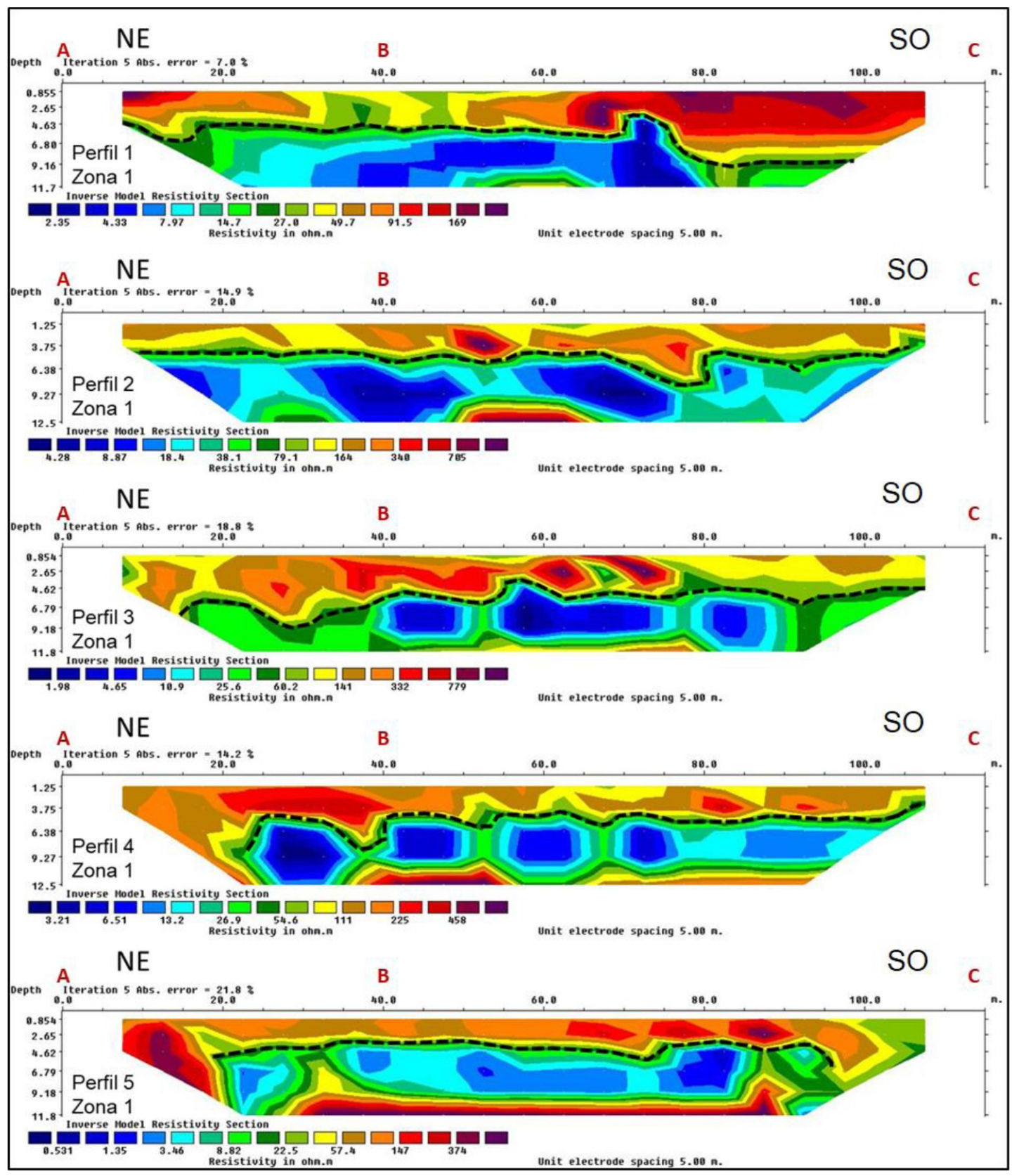

Figura 2. Perfiles de TRE adquiridos en la zona 1 con dirección NE-SO. Las líneas formadas por guiones de color negro indican el inicio de la zona de posible presencia de arenas saturadas con agua. La zona de pastizal está ubicada dentro de la longitud $A B$ y la zona de la palma de aceite se presenta en la longitud BC.

versión de los 5 perfiles de TRE de la zona que corresponde a la plantación de 25 años, obtenidos con dirección NE-SO, donde los primeros 30 metros de la parte NE fueron obtenidos sobre pastizales y el resto de los perfiles estaban posicionados sobre zona del cultivo de la palma de aceite. En los perfiles de esta zona, se registraron resistividades altas, que oscila entre 270 a $2000 \Omega \cdot m$, resistividades medias de entre $50-270 \Omega \cdot m$, y resistividades bajas, que oscilan entre 1 a $50 \Omega \cdot m$. Se observa un ligero incremento 


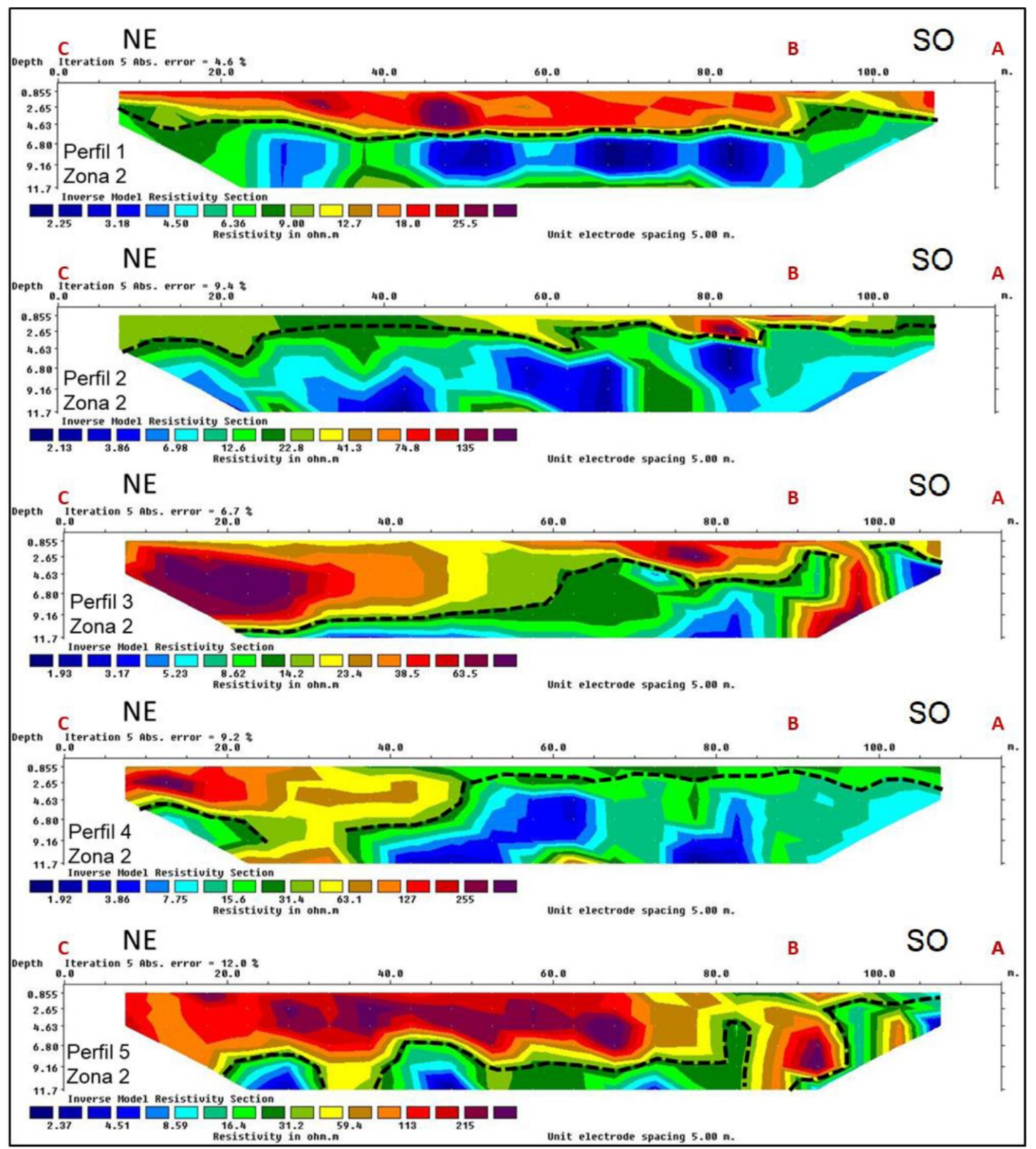

Figura 3. Perfiles de TRE adquiridos en la zona 2 con dirección NE-SO. Las líneas formadas por guiones de color negro indican el inicio de la zona de posible presencia de arenas saturadas con agua. La zona de pastizal está ubicada dentro de la longitud $A B$ y la zona de la palma de aceite se presenta en la longitud BC.

de la profundidad de la zona saturada, en dirección hacia donde se ubica el cultivo probablemente debido a la presencia de arenas saturadas con agua subterránea.

De manera general, se observa que las resistividades más bajas, que corresponden a los límites de las zonas saturadas, se encuentran en la zona 3 (plantación de 25 años), asimismo se nota que, en las zonas 2 (palmas de 5 años) y 3 (palmas de 25 años), la profundidad de la zona saturada es menor en el pastizal con respecto al cultivo de palma de aceite. 


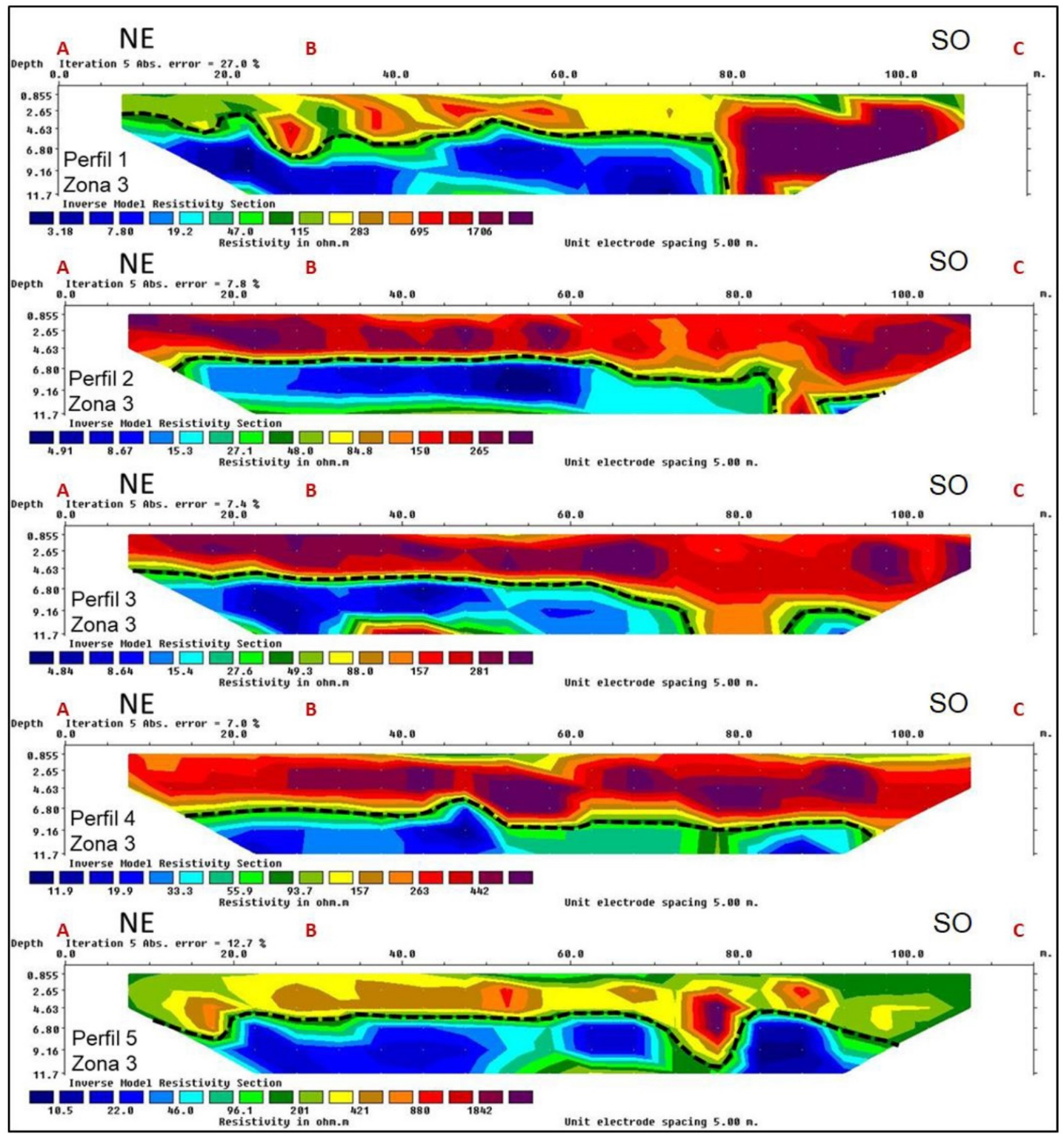

Figura 4. Perfiles de TRE adquiridos en la zona 3 (palma de aceite de 25 años) con dirección NE-SO. Las líneas formadas por guiones de color negro indican el inicio de la zona de posible presencia de arenas saturadas con agua. La zona de pastizal está ubicada dentro de la longitud AB y la zona de la palma de aceite se presenta en la longitud BC.

\section{DISCUSIÓN}

Las resistividades eléctricas son contrastantes en las tres zonas de cultivo, particularmente la zona 3 , con respecto a las zonas 1 y 2 respectivamente. Pero el patrón de RR es similar entre las tres zonas de estudio, ya que, en todas las zonas, se observa un gradiente de profundidad de la zona saturada que va de mayor a menor, según la profundidad del suelo, excepto en algunas partes del perfil 5 de la zona 1. En las tres áreas de estudio se mantienen altas resistividades en la parte superficial, mismas que van decreciendo hasta llegar a una zona de saturación entre los 4 y $6 \mathrm{~m}$, caracterizadas por bajas resistividades. La heterogeneidad de los valores de resistividades eléctricas que se observan en el estudio, se asocia a la heterogeneidad de materiales que subya- 
cen en el subsuelo, así como al grado de saturación con agua de dichas partículas. Las rocas sedimentarias como las que se distribuyen en la zona de estudio, son altamente variables en porosidad, lo que provoca diferentes grados de humedecimiento, que resultan en diferentes condiciones de fracturamiento, y humedad, que se refleja en variabilidad de los niveles de resistividad eléctrica (Guha et al. 2017). Las capas arenosas superficiales se caracterizan por elevadas resistividades eléctricas, mayores a 1241 $\Omega \cdot m$, las resistividades eléctricas de 76-232 $\Omega \cdot m$, corresponden a capas intermedias, condicionadas principalmente, por aumento del grado de saturación de los suelos arcillo-arenosos con la profundidad, mientras que los sedimentos finos presentan bajas resistividades eléctricas, en promedios de $19 \Omega \cdot m$ (García y Sacasas, 2017). Las altas resistividades eléctricas (100 a $800 \Omega \cdot m$ ) observadas en la zona 1 de la plantación de 11 años, pueden asociarse a arenas, limos y gravas saturadas por agua, las resistividades medias (35 a $100 \Omega \cdot m$ ) a la presencia de aluviones, arenas $\mathrm{o}$ arcillas saturadas, y las resistividades bajas (1 a $35 \Omega \cdot m$ ) posiblemente con arena saturada. Según Gonzales et al. (2016), las resistividades mayores a $100 \Omega \cdot m$ corresponden a materiales gruesos como arenas o gravas, mientras que valores menores a ese nivel, se asocian a sedimentos arcillosos finos, particularmente saturados, característicos de los depósitos aluviales.

En la zona 2 correspondiente a la plantación de 5 años, los valores de resistividades mayores oscilan entre 140 y $260 \Omega \cdot m$, asociadas posiblemente a la presencia de arenas arcillosas, $o$ arcillas arenosas limos y gravas con contenido de agua derivado de las lluvias de temporada. En esta zona, las resistividades medias (35 a $140 \Omega \cdot m$ ) se asocian posiblemente a aluviones y arenas, las bajas ( 1 y $35 \Omega \cdot m$ ) a depósitos de arcillas, aluviones o agua de la capa saturada. Al respecto Loke (2000) señala que las arcillas presentan resistividades entre 1-100 $\Omega \cdot m$, los aluviones entre 10-800 $\Omega \cdot m$, y el agua dulce del acuífero varía entre 10-100 $\Omega \cdot \mathrm{m}$. Si se toman en cuenta los señalamientos de Zavala-Cruz et al. (2016), de que los paisajes geomorfológicos de lomeríos suaves y valles acumulativos como los que se estudiaron, se distribuyen suelos Alisoles, Acrisoles y Luvisoles, que se caracterizan por horizontes $B$ desarrollados, que presentan acumulaciones de arcillas, esto explica en parte, las resistividades medias que se presentan entre los 2 y $3 \mathrm{~m}$ de la sub superficie, y que pueden ser correspondientes con las acumulaciones aluviales de partículas finas como arcillas, limos o arenas finas.

En el suelo de la zona 3 donde se establece la plantación de 25 años, las altas resistividades de entre 270 y $2000 \Omega \cdot m$, las medias de 50 a $270 \Omega \cdot m$, y las bajas de 1 a $50 \Omega \cdot m$, todas ellas asociadas a materiales de aluvión, asociadas a la posible presencia de arenas, limos y gravas con contenido de agua derivado de las lluvias de temporada, pueden estar siendo afectadas por otros factores que influyen sobre la resistividad eléctrica del suelo. Como la porosidad, contenido de agua, estructura, forma de las partículas y orientación, distribución de tamaño de las partículas, CIC, composición de cationes de la solución del suelo, temperatura, entre otros (Friedman, 2005). Asimismo, el gradiente de mayor a menor RE que se observa a partir de la superficie del suelo, deja de manifiesto un frente de humedecimiento, que va de menor a mayor humedad conforme aumenta la profundidad. Luz et al. (2019), menciona que el contenido de agua $(\omega)$ está relacionado con los valores de resistividad $(\rho)$, contenidos bajos de agua producen altos valores de resistividad y contenidos mayores al óptimo o cercanos al óptimo, producen valores bajos de $R E$, como se manifiestan en este estudio. Las mediciones de RE detectaron un área de saturación a ocho metros de profundidad, que probablemente corresponden a características propias del acuífero, ya que independientemente de las condiciones de precipitación que prevalecieron durante la investigación, la CONAGUA (2020) reporta el nivel estático de un acuífero entre 4.0 a $7.0 \mathrm{~m}$ de profundidad. Pero a pesar de lo somero que se encuentra la zona de saturación, la TRE revela que el nivel superior de dicha zona, en la mayoría de los perfiles se hace ligeramente más profundo en las áreas cultivadas con palma de aceite, con respecto a las áreas de pastizales, excepto en los perfiles 4 y 5 de la zona 1, y en los perfiles 3 y 5 de la zona 2, respectivamente. Dicho comportamiento se puede 
observar en las tres zonas, aunque se aprecia con mayor claridad en el tratamiento de palmas de 25 años. Dado que el estudio se realizó en temporada de lluvias, la profundización del nivel de la zona saturada en las plantaciones de palma de aceite plantea dos escenarios, que pueden ser motivos de nuevos estudios, por una parte, se podría pensar que la cercanía a la superficie de la zona saturada, permite que el cultivo se abastezca mayormente del acuífero, y en menor cantidad del agua que se infiltra después de la lluvia, por lo que la alta RR que se observa cerca de la superficie del suelo, y refleja áreas de menor humedecimiento, puede ser motivo de una pobre infiltración y un mayor nivel escurrimiento.

\section{CONCLUSIONES}

Se confirma un acuífero somero en el área de estudio, que no rebasa los $8 \mathrm{~m}$ de profundidad en el límite superior de la zona saturada. Con las resistividades más altas en la superficie y las resistividades más bajas en las capas más profundas, que indican homogeneidad en el material parental del terreno de estudio. Las resistividades eléctricas corresponden a arenas, limos, aluviones, arcillas y gravas. Los valores de resistividad eléctrica medidos no se relacionan con el grado de saturación y la litología, por lo que en el futuro deberán realizarse estudios de geofísica más profundos que correlacionen las variables antes mencionadas en las zonas de interés. Se observa un ligero abatimiento en el nivel superior del área saturada del pastizal, por lo que, al entrar a las áreas cultivadas con palma de aceite, dicho nivel se hace más profundo, independientemente de la edad de la plantación, aunque se observa con mayor claridad en la plantación de 25 años de edad. En la temporada de lluvia, en las capas más someras del terreno cultivado con palma de aceite hay un abatimiento de humedad con respecto a los pastizales, en particular en plantaciones de mayor edad, lo que sugiere que las palmas se abastecen principalmente del agua proveniente de la precipitación, y en menor cantidad del agua proveniente del acuífero.

\section{LITERATURA CITADA}

Ah Tung PG, Yusoff MK, Majid NM, Joo GK, Huang GH (2009) Effect of N and K fertilizers on nutrient leaching and groundwater quality under mature oil palm in Sabah during the monsoon period. American Journal of Applied Sciences 6: 1788-1799.

Banabas M, Turner MA, Scotter DR, Nelson PN (2008) Losses of nitrogen fertiliser under oil palm in Papua New Guinea: 1. Water balance, and nitrogen in soil solution and runoff. Australian Journal of Soil Research 46: 332-339

Carr MKV (2011) The water relations and irrigation requirements of oil palm (Elaeis guineensis) Experimental Agriculture 47: 629-652.

Chia MY, Huang YF, Koo CH, Fung KF (2020) Recent advances in evapotranspiration estimation using artificial intelligence approaches with a focus on hybridization techniques-a review. Agronomy 10: 1-33. https://doi.org/10.3390/agronomy10010101.

CONAGUA (2020) Actualización de la disponibilidad media anual de agua del acuífero La sierra (2705), Estado de Tabasco. Comisión Nacional del Agua https://www.gob.mx/cms/uploads/attachment/file/103374/DR_ 2705.pdf. Fecha de consulta: 05 de febrero de 2020.

Ellis RG, Oldenburg DW (1994) Applied geophysical inversion. Geophysics Journal International 116: 5-11

Friedman SP (2005) Soil properties influencing apparent electrical conductivity: a review. Computers and Electronics in Agriculture 46: 45-70

García GJ, Sacasas LC (2017) Modelos de resistividad eléctrica en formaciones geológicas del sur de Cuba occidental. Minería y Geología 33: 128-143. 
Gonzales AA, Dahlin T, Barmen G, Rosberg JE (2016) Electrical resistivity tomography and induced polarization for mapping the subsurface of alluvial fans: A case study in Punata (Bolivia). Geosciences 6: 51. DOI: 10.3390/geosciences6040051.

Guha RD, Singh TN, Kodikara J, Das R (2017) Correlating the mechanical and physical properties with mode-I fracture toughness of rocks. Rock Mechanics and Rock Engineering 50: 1941-1946.

Isaac-Márquez R, Valladares JLS, Spencer AE, Arcipreste MEA, Aguilar MAA, Márquez API, González M CS (2016) Impactos sociales y ambientales de la palma de aceite: Perspectiva de los campesinos en Campeche, México. Journal of Latin American Geography 15: 123-146

Loke MH (2000) Electrical imaging surveys for environmental and engineering studies: A practical guide to 2-D and 3-D surveys. 68p. https://pages.mtu.edu/ ctyoung/lokesnotes/LOKENOTE.PDF. Fecha de consulta: 2 de febrero del 2020.

Loke MH, Chambers JE, Rucker DF, Kuras O, Wilkinson PB (2013) Recent developments in the direct-current geoelectrical imaging method. Journal of Applied Geophysics 95: 135-156

Luz MJC, Arreygue RJE, Chávez NC, Equihua ALN (2019) Medición de resistividad eléctrica de un suelo característico de Morelia, Michoacán, considerando el efecto de la compactación. Ciencia Nicolaita 77: 73-86.

Maldonado MF, Jasso MJ, Palma LDJ, Salgado GS, González HVA (2006) Dinámica de materia orgánica, P y K en suelos de sistemas agroforestales-cedro-plátano en Tabasco, México. Revista Fitotecnia Mexicana 29: 223-230.

Mingorría S (2018) Violence and visibility in oil palm and sugarcane conflicts: the case of Polochic Valley, Guatemala. The Journal of Peasant Studies 45: 1314-1340.

Niwas S, Tezkan B, Israil M (2011) Aquifer hydraulic conductivity estimation fromsurface geoelectrical measurements for Krauthausen test site, Germany. Hydrogeology Journal 19: 307-315

Noguchi S, Nik A, Yusop Z, Tani M, Sammori T (1997) Rainfall-runoff responses and roles of soil moisture variations to the response in tropical rain forest, bukit tarek, peninsular Malaysia. Journal Forest Research 2: $125-132$

Perdomo S, Ainchil JE, Kruse EE (2014) Hydraulic parameters estimation from well logging resistivity and geoelectrical measurements. Journal of Applied Geophysics 105: 50-58

Ruiz-Álvarez O, Arteaga-Ramírez R, Vázquez-Peña MA, Ontiveros-Capurata RE, López-López R (2012) Balance hídrico y clasificación climática del estado de Tabasco, México. Universidad y Ciencia 28: 1-14.

Salazar ARB (2018) El agua subterránea y su importancia socioambiental. Universitarios potosinos 227: 16-21.

Sellers C, Corbelle E, Buján S, Miranda D (2015) Morfología interpretativa de alta resolución con datos LIDAR en la cuenca del río Paute-Ecuador. Memorias y Boletines De La Universidad Del Azuay 1: 399 - 411.

SIAP (2018) Servicio de Información Agroalimentaria y Pesquera. Anuario Estadístico de la Producción Agrícola, año 2019. http://infosiap.siap.gob.mx/aagricola_siap_gb/icultivo/index.jsp. Fecha de consulta: 2 de julio 2021.

Slater L (2007) Near surface electrical characterization of hydraulic conductivity: from petrophysical properties to aquifer Geometries-A review. Survey in Geophysics 28: 169-197

Tejero-Andrade A, Cifuentes G, Chávez RE, López-González AE, Delgado-Solórzano C (2015) L-and CORNERarrays for 3D electric resistivity tomography: an alternative for geophysical surveys in urban zones. Near Surface Geophysics 13: 355-368 
Yusop Z, Hui CM, Garusu GJ, Katimon A (2008) Estimation of evapotranspiration in oil palm catchments by short-time period water-budget method. Malaysian Journal of Civil Engineering 20: 160-174

Zavala-Cruz J, Jiménez Ramírez R, Palma-López DJ, Bautista Zúñiga F, Gavi Reyes F (2016) Paisajes geomorfológicos: base para el levantamiento de suelos en Tabasco, México. Ecosistemas y Recursos Agropecuarios 3: $161-171$

Zencich SJ, Froend RH, Turner JV, Gailitis V (2002) Influence of groundwater depth on the seasonal sources of water accessed by Banksia tree species on a shallow, sandy coastal aquifer. Oecologia 131: 8-19

Zhang I, Dawes WR, Walker GR (2001) Response of mean annual evapotranspiration to vegetation changes at catchment scale. Water Resources Research 37: 701-708. 